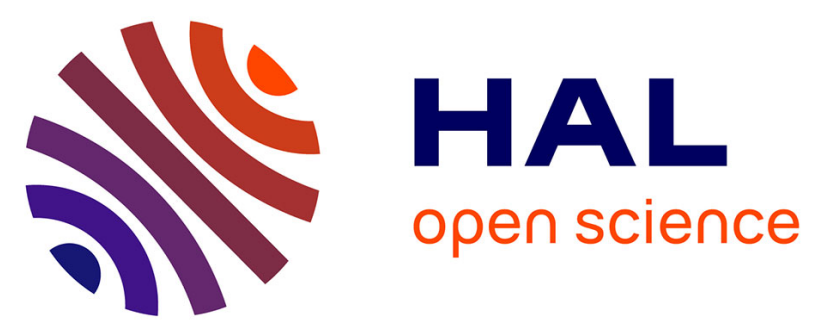

\title{
A 30 T pulsed magnet with conical bore for synchrotron powder diffraction
}

\author{
Julien Billette, Fabienne Duc, P. Frings, Marc Nardone, Abdelaziz Zitouni, \\ Carsten Detlefs, T. Roth, W. Crichton, Emilio Lorenzo, Rikken G.L.J.A.
}

\section{- To cite this version:}

Julien Billette, Fabienne Duc, P. Frings, Marc Nardone, Abdelaziz Zitouni, et al.. A 30 T pulsed magnet with conical bore for synchrotron powder diffraction. Review of Scientific Instruments, 2012, 83 (4), pp.043904. 10.1063/1.3701830 . hal-00976902

\section{HAL Id: hal-00976902 https://hal.science/hal-00976902}

Submitted on 14 Apr 2014

HAL is a multi-disciplinary open access archive for the deposit and dissemination of scientific research documents, whether they are published or not. The documents may come from teaching and research institutions in France or abroad, or from public or private research centers.
L'archive ouverte pluridisciplinaire HAL, est destinée au dépôt et à la diffusion de documents scientifiques de niveau recherche, publiés ou non, émanant des établissements d'enseignement et de recherche français ou étrangers, des laboratoires publics ou privés. 


\title{
A $30 \mathrm{~T}$ pulsed magnet with conical bore for synchrotron powder diffraction
}

\author{
J. Billette,${ }^{1}$ F. Duc, ${ }^{1}$ P. Frings, ${ }^{1}$ M. Nardone, ${ }^{1}$ A. Zitouni, ${ }^{1}$ C. Detlefs, ${ }^{2}$ \\ T. Roth, ${ }^{2}$ W. Crichton, ${ }^{2}$ J. E. Lorenzo, ${ }^{3}$ and G. L. J. A. Rikken ${ }^{1}$ \\ ${ }^{1}$ Laboratoire National des Champs Magnétiques Intenses, CNRS-INSA-UJF-UPS, 143, \\ avenue de Rangueil, F-31400 Toulouse, France \\ ${ }^{2}$ European Synchrotron Radiation Facility, Boite Postale 220, F-38043 Grenoble Cedex, France \\ ${ }^{3}$ Institut Néel, CNRS, Bô̂te Postale 166X, F-38043 Grenoble Cedex, France
}

(Received 1 February 2012; accepted 20 March 2012; published online 9 April 2012)

\begin{abstract}
We report on the design, construction, and operation of a horizontal field, $30 \mathrm{~T}$ magnet system with a conical bore optimized for synchrotron x-ray powder diffraction. The magnet offers $\pm 31^{\circ}$ optical access downstream of the sample, which allows to measure a sufficiently large number of Debye rings for an accurate crystal structure analysis. Combined with a $290 \mathrm{~kJ}$ generator, magnetic field pulses of $60 \mathrm{~ms}$ length were generated in the magnet, with a rise time of $4.1 \mathrm{~ms}$ and a repetition rate of 6 pulses/h at $30 \mathrm{~T}$. The coil is mounted inside a liquid nitrogen bath. A liquid helium flow cryostat reaches into the coil and allows sample temperature between 5 and $250 \mathrm{~K}$. The setup was used on the European Synchrotron Radiation Facility beamlines ID20 and ID06. (C) 2012 American Institute of Physics. [http://dx.doi.org/10.1063/1.3701830]
\end{abstract}

\section{INTRODUCTION}

Efforts to combine high magnetic fields with synchrotron $\mathrm{x}$-ray and neutron scattering experiments have given rise to the development of high field magnets with configurations other than simple solenoids. ${ }^{1-5}$ In scattering experiments both the direction of the incident and the exit beams must generally be varied relative to the sample. This requires either optical access over wide solid angles for both beams (with the sample fixed relative to the magnet), or wide optical access on the exit beam side only, while the sample is rotated within the magnet.

Two magnet geometries are possible:

- In the Faraday geometry the $\mathrm{x}$-ray or neutron beam travels through the bore of the magnet. The maximum angle between the incident (scattered) beam and the magnetic field is, therefore, limited by the diameter and length of the bore. This also limits the scattering angle $2 \theta_{B}$, i.e., the angle between the incident and scattered beams.

The Faraday geometry is best adapted for spectroscopy, e.g., $\mathrm{x}$-ray magnetic circular dichroism and magnetic Compton scattering, but it has also been used for diffraction experiments. ${ }^{6,7}$

- In Voigt geometry the incident and scattered beams are approximately perpendicular to the magnetic field axis. The magnet is implemented as two half magnets ("split-pair") arranged symmetrically above and below the mid plane. Split pair magnets offer the possibility to measure at large scattering angles within the midplane, but can accommodate only small angles out of this plane.

Superconducting magnets for $\mathrm{x}$-ray and neutron scattering are predominantly of the split-pair type, which at present limits the available magnetic field to $\approx 14-17 \mathrm{~T} .^{8-11}$ To date, most non-solenoid pulsed field magnets are also of the split-pair type. ${ }^{1-5}$
At the LNCMI-Toulouse (France) the first long pulse magnet system dedicated to synchrotron scattering experiments was designed around a "flat" solenoid coil. ${ }^{6}$ It has a short length compared to the inner diameter and thus the opening angle is increased up to $\pm 13^{\circ}$ compared to a classical solenoid coil. This coil was successfully used for synchrotron $\mathrm{X}$-ray powder diffraction measurements ${ }^{12}$ on the European Synchrotron Radiation Facility (ESRF) beamlines BM26 and ID20, and for x-ray absorption spectroscopy experiments ${ }^{13}$ on ID24. Encouraged by these results the design of a second magnet with possibilities for larger scattering angles was undertaken. The next natural step to increase as much as possible the opening angle in the Faraday geometry was the design of a conical bore magnet.

The conical configuration is particularly well adapted for powder diffraction coupled to a $2 \mathrm{D}$ detector. A detector located at a distance $L$ from the sample will be illuminated by a series of rings (Debye rings) of diameter, $D$, given by $D=2 L \tan \left(2 \theta_{B}\right)$. Each diffraction planes will be selected according to Bragg's law $\theta_{B}=\operatorname{asin}(\lambda / 2 d)$ where $\theta_{B}$ is the Bragg angle, $d$ is the spacing of the corresponding Bragg planes, and $\lambda$ is the photon wavelength.

In the conical configuration the signal can be recorded and radially integrated over the entire ring. Compared to a split-pair magnet in Voigt geometry, the number of diffracting grains (or powder average) is substantially increased, and the overall signal-to-noise ratio drastically improved.

Moreover, the conical geometry is a relatively safe and fast adaptation of a classical solenoid coil.

A $25 \mathrm{~T}$ steady-field conical magnet for neutron spectroscopy is presently under construction at the Helmholtz Center Berlin (Germany), ${ }^{14}$ in collaboration with the National High Magnetic Field Laboratory, Tallahassee (Florida, USA). ${ }^{15,16}$

Here, we present a horizontal field $30 \mathrm{~T}$ pulsed magnet specifically designed for synchrotron $\mathrm{x}$-ray powder diffraction. It offers the widest existing conical bore with a $\pm 31^{\circ}$ 
opening angle on the exit beam side. A detailed description of its design, test results and the integration of a cryogenic environment following the constraints of an $\mathrm{x}$-ray environment are reported.

\section{COIL DESIGN}

The coil is part of a system that includes a $290 \mathrm{~kJ}$ transportable generator ${ }^{6}$ and therefore its design has to be compatible with the features of the existing generator. This imposes constraints on the coil inductance, peak current and peak voltage, which in turn limits the choice of wire materials, overall coil size, pulse length, and peak magnetic field, etc.

The generator is based on a capacitor bank with a total capacitance of $1 \mathrm{mF}$ and a maximum charge voltage of $24 \mathrm{kV}$. The peak current of this generator is limited to about $30 \mathrm{kA}$ by inductive current limiters with a total inductance of $0.25 \mathrm{mH}$ and a resistance of $17.5 \mathrm{~m} \Omega$. It is also equipped with crowbar resistors of $225 \mathrm{~m} \Omega$ that can modify the decay of the magnetic field pulse to reduce coil heating and avoid reverse voltages on the thyristors and capacitors.

One of the first choices made for the new coil was to limit the maximum field to $30 \mathrm{~T}$ to ensure a long lifetime and reliability as well as to minimize safety issues of the equipment. Other constraints that had to be respected concerned the pulse duration which has to be long enough to allow x-ray exposure times of $\approx 2 \mathrm{~ms}$ duration. This time constraint is given by a mechanical $x$-ray shutter and allows to collect diffraction images of adequate exposure without repeating shots. ${ }^{17}$

We decided to build similar cryogenic environments as for the previous coil $^{6}$ with a nitrogen bath to cool the magnet and the possibility to regulate the sample temperature over a wide range $(5-250 \mathrm{~K})$ by means of a helium flow cryostat. This implied that the bore size was determined by the required optical access size and the different isolation vacuum and cryogenic components.

To preserve the mechanical load symmetry, and therefore the stability of the system, it was decided to have a conical winding on both sides of the midplane even if the wide optical access was only needed downstream of the sample. These design constraints lead to a coil model with a bore diameter of $20 \mathrm{~mm}$, an outer diameter of $130 \mathrm{~mm}$, a length of $80 \mathrm{~mm}$, and an opening angle of $\pm 40^{\circ}$ at both ends.

$\mathrm{A} \mathrm{Cu}$ wire reinforced with $\mathrm{NbTi}$ filaments was selected. This material combines high conductivity (low heating) with high mechanical strength (see Table I).

In order to achieve the long-term reliability necessary for user experiments, the design of the coil was optimized using finite-element analysis (FEA). The commercial code COMSOL, which consists of general "Multiphysics" packages, was used to take into account the electrical, thermal, and mechanical loads. For the finite-element model, the coil was assumed to have axial symmetry and to be symmetric with respect to the midplane, omitting thereby the helicity of the coil and the layer transitions. The problem was thus simplified to a twodimensional one and the calculations were performed over a quarter of the cross section of the coil.

The resulting stress and strain distributions in the midplane of the coil calculated for a magnetic field of $35 \mathrm{~T}$ are dis-
TABLE I. Mechanical and electrical properties of the wire conductor. The copper wire was reinforced by $630 \mathrm{NbTi}$ filaments of around $82 \mu \mathrm{m}$ diameter. The volume fraction of the $\mathrm{NbTi}$ determined from the number of filaments and their average diameter is around $51 \%$.

\begin{tabular}{ll}
\hline \hline Young modulus & $\mathrm{E}=105 \mathrm{GPa}$ \\
Elastic stress limit & $\sigma_{y s}(300 \mathrm{~K})=400 \mathrm{MPa}$ \\
& $\sigma_{y s}(77 \mathrm{~K})=650 \mathrm{MPa}$ \\
Isotropic plastic modulus & $\mathrm{E}_{t i s o}=166 \mathrm{MPa}$ \\
Ultimate tensile strength & $\mathrm{UTS}_{77 \mathrm{~K}}=710 \mathrm{MPa}$ \\
Ultimate plastic strain & $\varepsilon_{u 77 \mathrm{~K}}=30 \%$ \\
Resistivity & $\rho_{300 \mathrm{~K}}=2.59 \mu \Omega . \mathrm{cm}$ \\
& $\rho_{77 \mathrm{~K}}=0.32 \mu \Omega . \mathrm{cm}$ \\
Cross section & $\mathrm{S}=6.43 \mathrm{~mm}^{2}$ \\
Thickness $\times$ width & $=2.23 \times 3.13 \mathrm{~mm}$ \\
\hline \hline
\end{tabular}

played in Fig. 1. The von Mises stress is the criterion for mechanical failure related to plastic deformation. Here, it originates mainly from the stress component tangential to the wire (hoop stress) which is caused by the axial component of the magnetic field. Its maximum value is around $650 \mathrm{MPa}$, which is close to the ultimate tensile strength of the conductor (710 $\mathrm{MPa})$. Since stresses scale as the square of the magnetic field we have a sufficient safety margin for testing at $32 \mathrm{~T}$ (545 $\mathrm{MPa})$ and routine operation up to $30 \mathrm{~T}(480 \mathrm{MPa})$.

The strain value determines the degree of the plastic deformation of the wire. In our case, we observe a tendency to layer separation up to the 7 th layer. Since the deformations scale similarly and will thus only be $73 \%$ at $32 \mathrm{~T}$ we do not

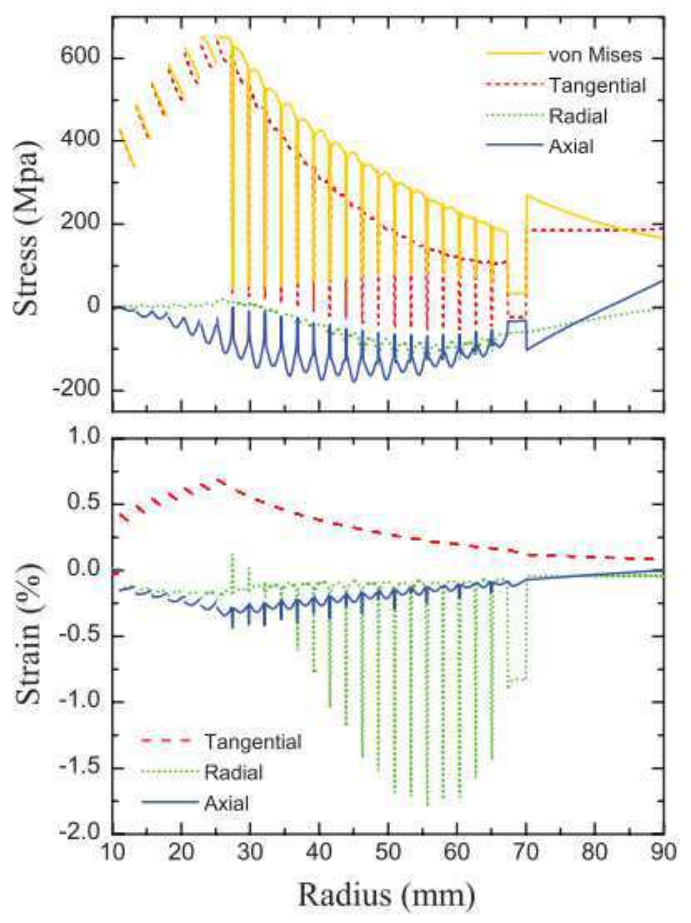

FIG. 1. Calculated stress (top)/strain (bottom) distributions in the midplane of the coil at $35 \mathrm{~T}$. From 10 to $67 \mathrm{~mm}$, the stress/strain are calculated along the 24 layers of the coil, then along the insulation layer of glass fiber and epoxy resin (up to $70 \mathrm{~mm}$ ) and finally along the external stainless steel reinforcement (beyond $70 \mathrm{~mm}$ ). 


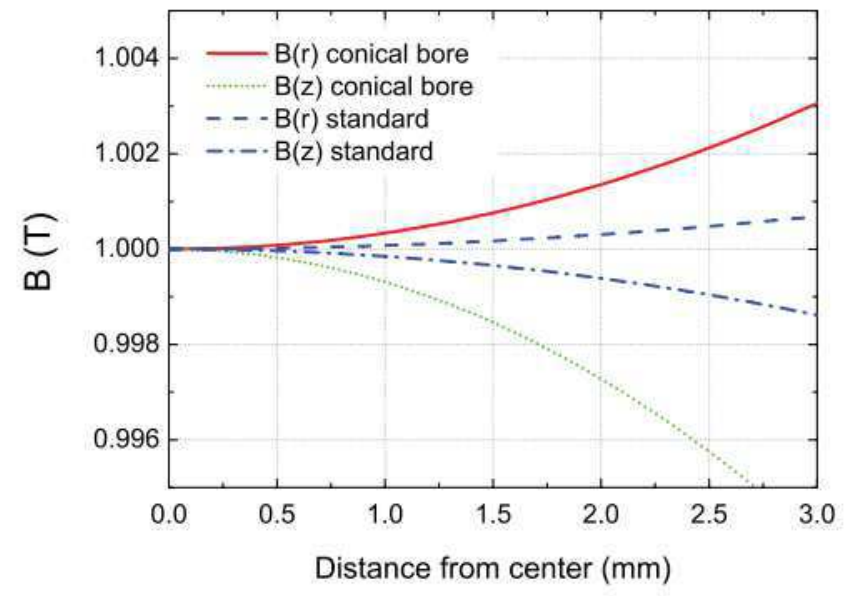

FIG. 2. Magnetic field homogeneity from the center of the magnet calculated for a magnet with conical ends compared to a classical solenoid magnet. The axial and radial distribution are denoted by $\mathrm{B}(\mathrm{z})$ and $\mathrm{B}(\mathrm{r})$, respectively. The magnetic field at the center of the coil was normalized to $1 \mathrm{~T}$.

expect any performance degradation/stability issues because of excessive strain.

The electrical parameters and the thermal load the magnet will be subjected to during the field pulse were also evaluated by finite-element analysis. The values thus simulated confirmed the feasibility of the coil and were in close agreement with the actual values measured once the coil was wound.

Finally, the field distributions inside a conical bore magnet and inside a classical solenoid coil were computed and compared, as shown in Fig. 2. Despite the reduction of the magnetic field homogeneity due to conical winding, homogeneity over a typical powder sample (i.e., sample diameter $\emptyset \approx 4 \mathrm{~mm}$, sample thickness $\mathrm{t}=100 \mu \mathrm{m})^{18}$ is better than $1.5 / 1000$.

\section{CONSTRUCTION AND OPERATION}

\section{A. Coil construction}

The conical winding consists of a progressive increase of the layer length in the inner half part of the coil. Accordingly, the most critical part of the coil winding lies in the regular- ity of the transition between the layers to keep an excellent alignment of outer layers which are longer than inner ones.

To make the winding easier, the wire was wound onto a coil-body of glass fiber epoxy composite (FR4/G10) machined such that the winding length was progressively increased, thus widening conically the bore at both ends of the coil, as shown in Fig. 3. The winding was completed with a $5 \mathrm{~mm}$ thick insulation layer of glass fiber impregnated with epoxy resin. For the external reinforcement, a stainless steel cylinder was shrunk onto the coil. G10 flanges were bolted onto both ends of this cylinder. These were used to mount the coil in the bottom of the liquid nitrogen cryostat.

For powder diffraction, optical access for the incident $\mathrm{x}$-ray beam is required only over a narrow solid angle. The electrical connections were therefore located on this side of the coil (see Fig. 4). The transition from the windings to the connectors were made such that the wire is bent as little as possible.

\section{B. Commissioning}

A simple liquid nitrogen (LN2) bath cryostat was used to evaluate the maximum field and repetition rate. The field was measured using a pick-up coil in the center of the pulsed coil. The resistance of the coil was continuously monitored during cooling after each pulse. The coil parameters as measured during coil testing were: a resistance value of $R_{\text {coil }}=60.55 \mathrm{~m} \Omega$ and $485 \mathrm{~m} \Omega$ at $77 \mathrm{~K}$ and $300 \mathrm{~K}$, respectively, an inductance $l$ $=8.199 \mathrm{mH}$ and a field factor of $176.8 \mathrm{~A} / \mathrm{T}$.

The cooling time of this magnet depends on the operating temperature and the energy deposited in the coil during the pulse, i.e., the field strength. At higher operating temperatures, heat is transferred faster to the LN2 bath, but the electrical resistance of the coil is higher (leading to more deposited energy at equal field strength), and the mechanical strength is reduced.

Typical pulses are shown in Fig. 5. During testing, the magnet has achieved a maximum magnetic field of $31.8 \mathrm{~T}$ for a charging voltage of $V=20 \mathrm{kV}$. The field rises from zero to maximum within $4.1 \mathrm{~ms}$. This pulse was started with the coil at liquid nitrogen temperature. After the pulse, the final temperature of the coil was $109 \mathrm{~K}\left(R_{\text {coil }} \approx 121 \mathrm{~m} \Omega\right)$. A series of pulses were then performed at the maximum
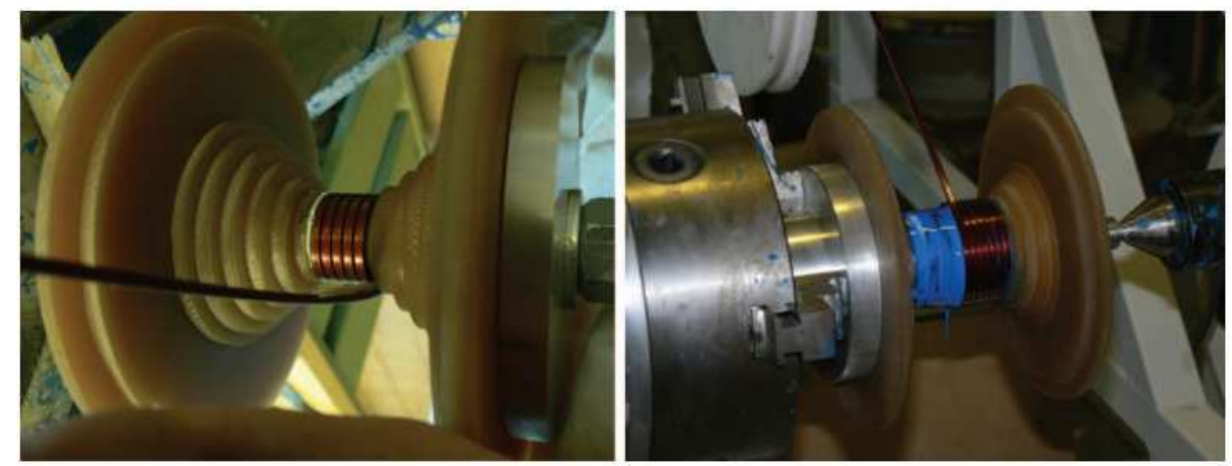

FIG. 3. (Left) Beginning of the winding showing the special G10 coil-body used to allow the increase of the length of the subsequent layers. (Right) Between each layer, the conductor was wetted with Stycast 2850 blue epoxy. 


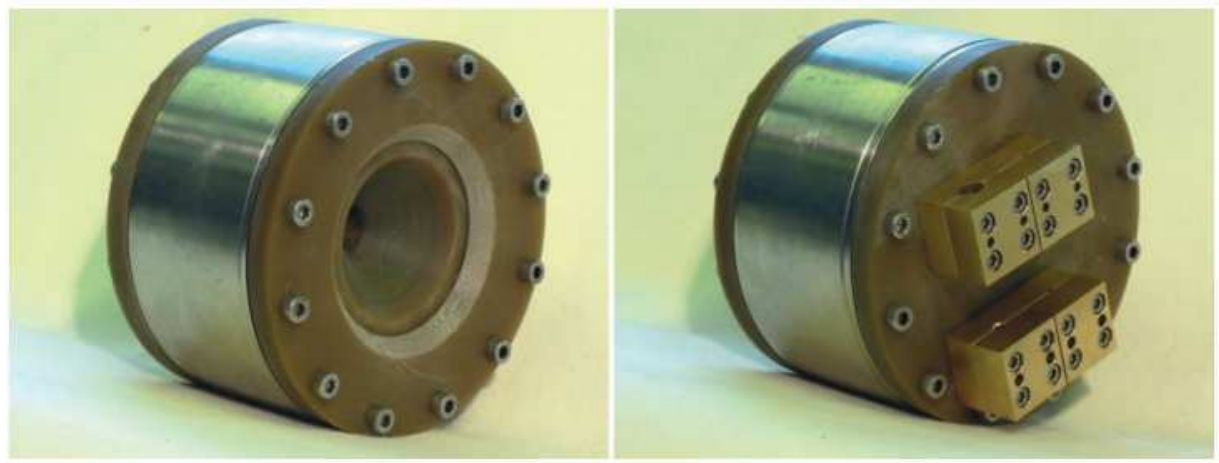

FIG. 4. Coil view from the wide angle side (left), from the connectors side (right).

operating field value of $30 \mathrm{~T}$ (charge voltage $19 \mathrm{kV}$ ) to evaluate the repetition rate. From these test results, the maximum operating temperature was chosen at $100 \mathrm{~K}\left(R_{\text {coil }}=98 \mathrm{~m} \Omega\right)$. With this choice, during a $30 \mathrm{~T}$ pulse, the coil heats from $100 \mathrm{~K}$ to $125 \mathrm{~K}$, and the resistance of the coil varies from 98 to $155 \mathrm{~m} \Omega$. The cooling time to return to $100 \mathrm{~K}$, shown in Fig. 6, is less than $9 \mathrm{~min}$, thus a repetition rate of up to 6 shots per hour can be sustained. The maximum repetition rate at lower fields scales approximately as $1 / B_{\text {peak }}^{2} \propto 1 / V^{2}$ as the energy deposited through resistive heating is given by $\int I^{2}(t) R_{\text {coil }}(t) \mathrm{d} t \approx$ const. $\cdot B_{\text {peak }}^{2} \cdot\left\langle R_{\text {coil }}\right\rangle$.

Combined with the high flux provided by an undulator station, the duty cycle of this system is sufficient for accurate structural analysis. Experiments carried out on the ESRF beamlines ID20 and ID06 recorded sufficient statistics within one single shot. ${ }^{20}$

\section{INTEGRATION INTO A CRYOGENIC ENVIRONMENT}

The optical access along a horizontal axis upstream and downstream of the sample required by the x-ray beam imposed a common design for the coil and cryostat. Each component was designed as a part of an integrated system.

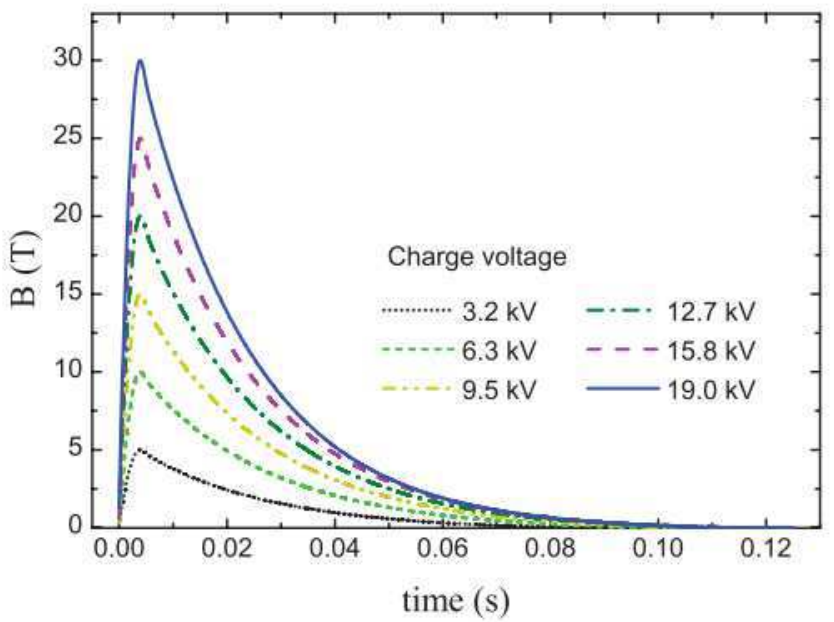

FIG. 5. Pulse field shape as measured at different charge voltages. The asymmetry of the pulse shape is due to the relatively high crowbar resistance. ${ }^{19}$
The cryogenic system was designed to meet the following requirements:

- Cooling of the coil by direct contact with liquid nitrogen.

- X-ray beam passage in vacuum $<10^{-4} \mathrm{mbar}$, through both the coil and the bore of the helium cryostat.

- Sample in vacuum.

- Maximum opening angle on the downstream side of the cryostat (as close as possible of the opening angle allowed by the coil).

- Quick dismounting for a possible replacement of the coil and the coaxial current leads.

- Variable temperature over the range 5-250 K.

- Quick sample loading without venting of the vacuum.

The system (see Fig. 7) is composed of three distinct subassemblies: the nitrogen cryostat, the helium flow cryostat, and the sample handling system.

\section{A. Nitrogen cryostat}

The liquid nitrogen (LN2) cryostat (Fig. 7, black) is a double-walled bath with an isolation vacuum. A cylindrical tube (on the upstream side) traverses the coil along the center of the LN2 cryostat. It is welded onto a cone (on the

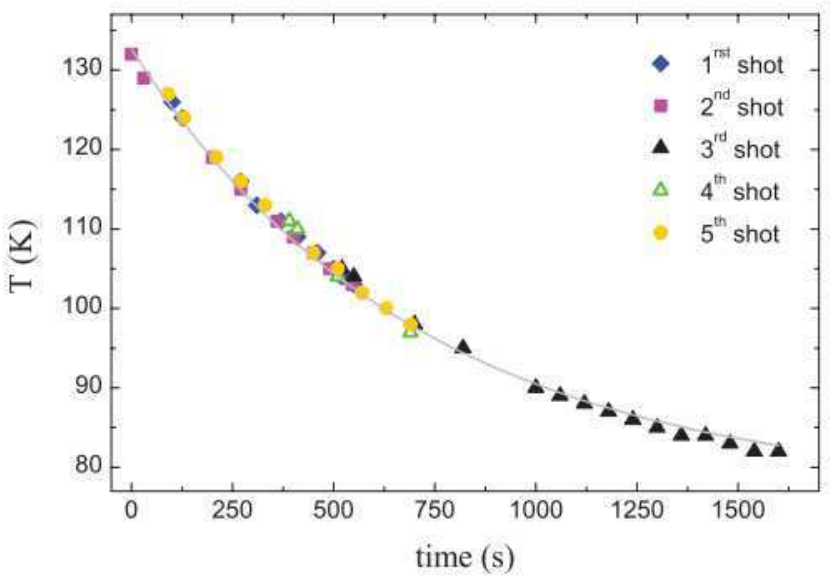

FIG. 6. Cooling time for a series of shots at charge voltage of $19 \mathrm{kV}$ (giving about $30 \mathrm{~T}$ peak fields). These shots were carried out immediately after a first shot at the maximum charge voltage of $20 \mathrm{kV}$. The solid line was obtained by fitting the data to an exponential decay function $\left(T(K)=T_{0}+\Delta T \exp (-t / \tau)\right.$ with $\tau=706( \pm 10) \mathrm{s}$, and $\left.\Delta T=56( \pm 1) \mathrm{K}\right)$. 


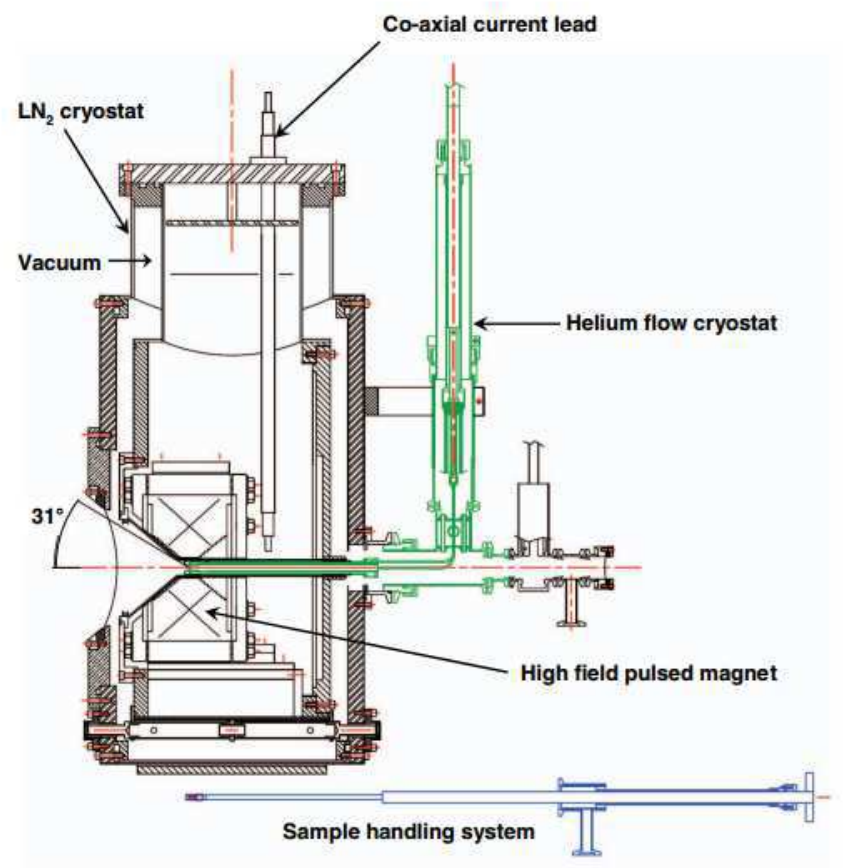

FIG. 7. Cross-section of the magnet/liquid nitrogen bath/sample cryostat assembly. The $\pm 40^{\circ}$ opening angle is given for a typical sample (i.e., sample diameter $\emptyset \approx 4 \mathrm{~mm}$, sample thickness $\mathrm{t}=100 \mu \mathrm{m}$ ) at the center of the coil without cryogenic environment. Once the coil integrated into the LN2 cryostat, the effective angle available for $x$-ray scattering is reduced to $\pm 31^{\circ}$.

downstream side) to provide an in-vacuum cold bore into which the helium cryostat and sample can be inserted. The mounting interface for the helium cryostat on the upstream side is a KF50 flange. The exit cone is closed by a demountable, $\mathrm{x}$-ray transparent Kapton window $(100 \mu \mathrm{m})$. Once the coil was integrated into the LN2 cryostat, the effective angle available for $x$-ray scattering was reduced to $\pm 31^{\circ}$, which is still the widest existing optical access for a pulsed field magnet in the Faraday geometry.

The coaxial current lead, LN2 filling port, venting port, level gauge, etc., are mounted on a polycarbonate flange at the top of the assembly.

\section{B. Helium flow cryostat}

The helium flow cryostat (Fig. 7, green) is inserted into the coil bore from the upstream side, based on the same principle as our first design. ${ }^{6,12}$ It consists of a vertical and a horizontal part.

A Oxford Instruments Low loss transfer tube (LLTT) is used to inject liquid ${ }^{4} \mathrm{He}$ (LH4) into the circuit, and to recover the exhaust gas. The flow rate of $\mathrm{LH} 4$ can be adjusted by a needle valve on the LLTT. Additionally, a resistive heater can be used to warm the passing gas before it is transferred towards the sample position in the center of the magnet via a capillary.

The horizontal part of the LH4 cryostat consists mainly of two concentric hollow tubes inserted into the bore of the magnet. The He gas circulates between the tubes. The sample can be mounted on a threaded section inside the inner tube near the center of the magnet. Fiber glass spacers and wedges stabilize the position of the cryostat. The cryostat is made almost entirely of $304 \mathrm{~L}$ grade stainless steel, as a compromise between thermal conductivity and high electrical resistivity to avoid currents induced by the field pulse.

The LN2 and LH4 cryostats share the same insulation vacuum, and the $\mathrm{LN} 2$ bath acts as a $77 \mathrm{~K}$ radiation screen for large parts of the LH4 cryostat.

\section{Sample handling system}

The sample handling system (Fig. 7, blue) is a load air lock connected to a gate valve. When open, a transfer rod is inserted into the vacuum, through the inner bore of the horizontal helium cryostat, up to the center of the coil.

At the extremity of the rod, the sample holder is mounted on a left-handed thread (Fig. 7, purple). The sample holder is a stainless steel hollow cylinder threaded at half length of its downstream outer side, and with a left-handed thread on the inside upstream side. The sample, usually a compressed pellet, is glued inside the downstream end of the cylinder. After screwing the sample holder into the operating position, the transfer rod is unscrewed and pulled out of the vacuum up to the gate valve. The gate valve is then closed so that the air lock can be dismounted from its KF25 flange and be replaced by a Kapton window on a KF25 flange. After pumping the volume inside the connection flanges, the gate valve is opened to allow the x-ray beam to access up to the sample. Typically, sample changes take less than $10 \mathrm{~min}$.

The sample cooling is achieved by thermal contact between the sample holder and the heat exchanger of the helium cryostat. During preliminary tests performed without beam, a stable $5 \mathrm{~K}$ temperature was measured with a calibrated Cernox sensor glued at the sample position.

\section{EXAMPLES OF SCIENTIFIC APPLICATIONS}

Commission tests of the new coil were carried out at beamline ID06, ESRF. Synthetic powder samples of high $T_{c} \mathrm{NdFeAsO}$ and of natural atacamite, $\mathrm{Cu}_{2} \mathrm{Cl}(\mathrm{OH})_{3}$, have been measured in pulsed fields of up to $30 \mathrm{~T}$, using pulsesimultaneous exposure times of less than $10 \mathrm{~ms}$ at temperatures down to $70 \mathrm{~K}(\mathrm{NdFeAsO})$ and $6 \mathrm{~K}$ (atacamite). Experimental conditions and configuration were those previously described in detail in Refs. 6 and 20.

The two samples offer different challenges; not only from a point of view of disturbing their inherent magnetic properties at reduced temperature, but also from our ability to extract reliable structural information from powder samples with contrasting symmetries and structural complexities. In both cases, data were collected on a mar345 image plate detector with diffraction limited to $2 \theta \sim 18^{\circ}$, or $2 d>1.25 \AA$ at $33 \mathrm{keV}$ incident $\mathrm{x}$-ray energy. Exposure times were pulselocked via a TTL-triggered ms fast shutter.

\section{A. $\mathrm{NdFeAsO}$}

For NdFeAsO, it is evident from Fig. 8 that lattice distortion markers show most variance in the range of temperatures between 120 and $205 \mathrm{~K}$, i.e., about the 


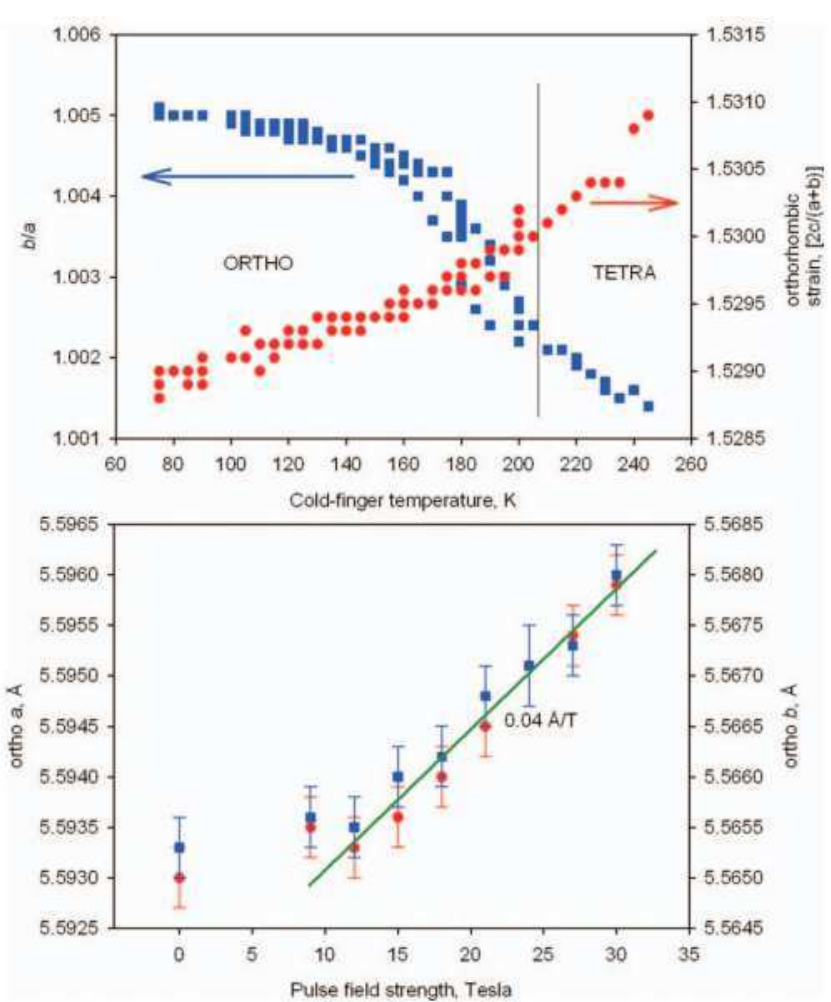

FIG. 8. Lattice distortion in NdFeAsO. The top panel shows the variation in $b / a$, or the level of in-plane orthorhombic distortion (blue squares) and the level of pseudo-tetragonal $[2 c /(a+b)]$ distortion (red circles) at all temperatures and fields of 3-30 T. The lower panel indicates the effect on in-plane cell parameters at a fixed temperature of $90 \mathrm{~K}$ while augmenting pulse strength to $30 \mathrm{~T}$. The green line shown indicates an increasing trend of $0.04 \AA \mathrm{T}^{-1}$ orthorhombic-tetragonal transition temperature. They further show a clear hysteresis envelope about the transition temperature that delimits data from all field strengths; we are therefore able to gauge an effect of likely lattice strain induced under high-field conditions. A subset of these data, extracted for a single temperature point, are shown in the lower panel and demonstrate that a field-dependent structural response is evident in measurements of the in-plane lattice directions, and that this effect is not saturated by application of $30 \mathrm{~T}$. The key point with this test material remained to determine if the subtle group-subgroup transition could be observed, i.e., is there sufficient resolution to observe tetragonal-orthorhombic peak-splitting, and to what degree lattice response was affected, and measurable, with reasonable certainly. It is clear that we were successful (all refinements were of similar quality, with refined $R p \sim 0.06-0.07$ ).

\section{B. $\mathrm{Cu}_{2} \mathrm{Cl}(\mathrm{OH})_{3}$}

The tests of atacamite were primarily to identify if it was prudent to collect data on a altogether more complicated orthorhombic structure with a lower scattering power than the rare-earth-containing pnictide above.

This was further complicated by data collections at base temperature, in an otherwise identical diffraction setup. The eventual aim would be to determine internal structural parameters in order to estimate if it would be possible to extract any effect of high-field application on the frustration of the Kagomé-like lattice. The first stage of this endeavour is nonetheless to demonstrate that we can be successful in refining atacamite with reasonable precision, to $R_{\text {Bragg }}$ of 0.06 , at sub $10 \mathrm{~K}$, with field strengths of up to $30 \mathrm{~T}$ and pulselocked exposure times. Figure 9 shows the refined structure,

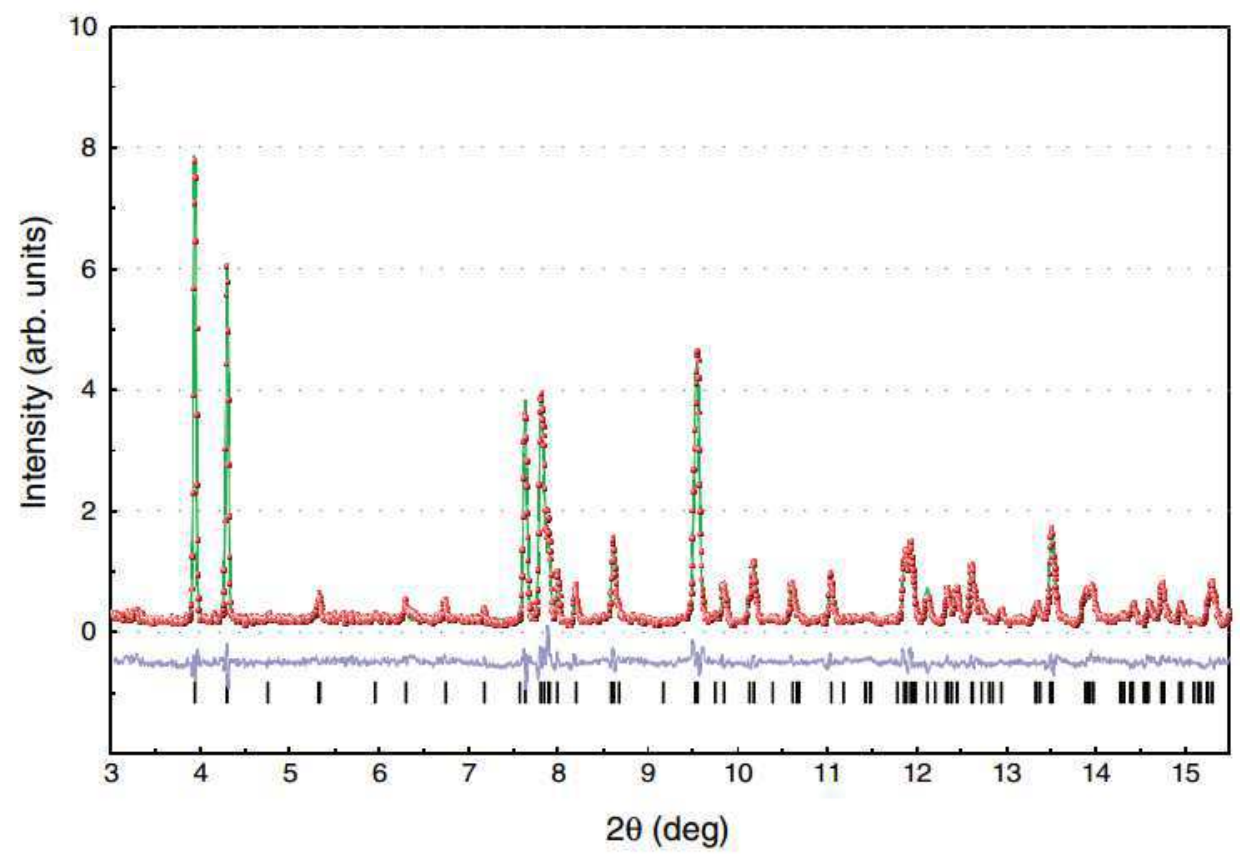

FIG. 9. Final Rietveld plot $\left(R_{\mathrm{Bragg}}=0.06\right)$ for the structure of atacamite $(P n m a, \mathrm{a}=6.030(2), \mathrm{b}=6.865(2)$, and $\mathrm{c}=9.12(2) \AA)$ at $\mathrm{T}=7 \mathrm{~K}, \mathrm{~B}=30 \mathrm{~T}$ from $7 \mathrm{~ms}$ data collection on mar345 image plate. The experimental data are represented by red dots, while the fitted pattern is given by the solid green line. The vertical lines are the position of the Bragg peaks calculated by Rietveld refinement. The lower trace corresponds to the difference between the observed and the calculated profile. 
indicating that a more comprehensive survey of temperaturefield space could determine any field effect on these frustrated systems.

We are confident therefore in these preliminary test results. In both cases bulk structural response to high field at variable temperature is measurable with single-shot exposures. Additionally, not only were we able to obtain lattice parameters as a function of temperature and field but we were also able to refine internal parameters, this opens the door to study; for example, site-specific structural responses. Future upgrades; such as faster, higher resolution pixelated detectors can only improve this situation.

\section{CONCLUSIONS AND FUTURE OUTLOOK}

In summary, we have constructed a pulsed magnet and cryostat system optimized for x-ray powder diffraction at synchrotron sources. The magnet has a novel bi-conical geometry that allows $\mathrm{x}$-ray optical access over a wide range of scattering angles, $\pm 31^{\circ}$ downstream of the sample. Using a capacitive generator, the magnet can be operated up to $30 \mathrm{~T}$ with a high repetition rate of up to 6 pulses per hour at maximum field. Up to now, more than seven hundred pulses, half of them being done at $30 \mathrm{~T}$, have been performed using this setup without sign of degradation of the coil. The FEA modeling suggests that the magnet should have an operational life-time of several thousand $30 \mathrm{~T}$ pulses.

The present design is rather versatile. It can be further improved, for instance, by incorporating LN2 cooling channels that decrease the cooling time of the coil and thus improve the duty cycle.

Based on the experience gained during the design, construction, and operation of this magnet we will develop a similar system optimized for neutron diffraction and spectroscopy.

\section{ACKNOWLEDGMENTS}

The authors would like to thank F. Lecouturier and N. Ferreira of the LNCMI/CNRS for the characterization of the wire conductor (resistivity and wire strength measurements). The samples of $\mathrm{NdFeAsO}$ were kindly provided by S. Margadonna. The authors acknowledge the ESRF for granting the beam time for these experiments. The safety issues of the equipment were addressed with the help of ESRF Safety Group. The MAR345 online image plate detectors were lent to the authors by the ESRF detector pool. Part of this research was funded by the ANR (Grant No. ANR-05-BLAN-0238).
${ }^{1}$ Y. H. Matsuda, Y. Ueda, H. Nojiri, T. Takahashi, T. Inami, K. Ohwada, Y. Murakami, and T. Arima, Physica B 346, 519 (2004).

${ }^{2}$ Y. Narumi, K. Kindo, K. Katsumata, M. Kawauchi, C. Broennimann, U. Staub, H. Toyokawa, Y. Tanaka, K. Kikkawa, T. Yamamoto, M. Hagiwara, T. Ishikawa, and H. Kitamura, J. Phys.: Conf. Ser. 51, 494 (2006).

${ }^{3}$ Y. Narumi, K. Kindo, K. Katsumata, M. Kawauchi, C. Broennimann, U. Staub, H. Toyokawa, Y. Tanaka, A. Kikkawa, T. Yamamoto, M. Hagiwara, T. Ishikawa, and H. Kitamura, J. Synchrotron Radiat. 13, 271 (2006).

${ }^{4}$ Y. H. Matsuda, T. Inami, K. Ohwada, Y. Murata, H. Nojiri, Y. Murakami, H. Ohta, W. Zhang, and K. Yoshimura, J. Phys. Soc. Jpn. 75, 024710 (2006).

${ }^{5}$ Z. Islam, J. P. C. Ruff, H. Nojiri, Y. H. Matsuda, K. A. Ross, B. D. Gaulin, Z. Qu, and J. C. Lang, Rev. Sci. Instrum. 80, 113902 (2009).

${ }^{6}$ P. Frings, J. Vanacken, C. Detlefs, F. Duc, J. E. Lorenzo, M. Nardone, J. Billette, A. Zitouni, W. Bras, and G. L. J. A. Rikken, Rev. Sci. Instrum. 77, 063903 (2006)

${ }^{7}$ Z. Islam, D. Capatina, J. P. C. Ruff, R. K. Das, E. Trakhtenberg, H. Nojiri, Y. Narumi, U. Welp, and P. C. Canfield, Rev. Sci. Instrum. 83, 035101 (2012).

${ }^{8}$ M. Meissner and P. Smeibidl, Neutron News 12, 12 (2001).

${ }^{9}$ K. Prokes, M. Meissner, P. Smeibidl, C. Fritsche, K.-D. Ohloff, and P. Daniels, Physica B 294-295, 691 (2001).

${ }^{10}$ L. Paolasini, C. Detlefs, C. Mazzoli, S. Wilkins, P. P. Deen, A. Bombardi, N. Kernavanois, F. De Bergevin, F. Yakhou, J. P. Valade, I. Breslavetz, A. Fondacaro, G. Pepellin, and P. Bernard, J. Synchrotron Radiat. 14, 301 (2007).

${ }^{11}$ C. S. Nelson, H. Mo, B. Bohnenbuck, J. Strempfer, N. Kikugawa, S. I. Ikeda, and Y. Yoshida, Phys. Rev. B 75, 212403 (2007).

${ }^{12}$ C. Detlefs, F. Duc, Z. A. Kazei, J. Vanacken, P. Frings, W. Bras, J. E. Lorenzo, P. C. Canfield, and G. L. J. A. Rikken, Phys. Rev. Lett. 100, 056405 (2008).

${ }^{13}$ J. Vanacken, C. Detlefs, O. Mathon, P. Frings, F. Duc, J. E. Lorenzo, M. Nardone, J. Billette, A. Zitouni, M. C. Dominguez, J. Herczeg, W. Brass, V. V. Moshchalkov, and G. Rikken, AIP Conf. Proc. 902, 103 (2007).

${ }^{14}$ M. Steiner, D. A. Tennant, and P. Smeibidl, J. Phys.: Conf. Ser. 51, 470 (2006).

${ }^{15}$ M. D. Bird, S. Bole, S. Gundlach, and J. Toth, IEEE Trans. Appl. Supercond. 16, 957 (2006).

${ }^{16} \mathrm{~J}$. P. Chen, M. D. Bird, S. Bole, S. Gundlach, J. O'Reilly, and Y. L. Viouchkov, IEEE Trans. Appl. Supercond. 20, 636 (2010).

${ }^{17}$ The mechanical shutter was the model LS200 Laser shutter from NM Laser products, Inc. (San Jose, CA, USA). On the basis of the pulse shape obtained with the features of the generator mentioned above, the range of field experienced by a sample for a $30 \mathrm{~T}$ pulse during a minimum exposure of $\sim 2 \mathrm{~ms}$ was between 28.5 and $30 \mathrm{~T}$.

${ }^{18}$ Samples are typically compressed pellets of polycrystalline samples. They are prepared to fit onto the sample holder which is a stainless steel hollow cylinder of $4 \mathrm{~mm}$ inner diameter and $18 \mathrm{~mm}$ in length. The powder pellets are embedded into a low molecular weight resin (polyvinylpyrolidone) in order to prevent grain movement and glued with the same resin inside the downstream end of the cylinder. The pellet thickness is adjusted to about one absorption length at the working energy.

${ }^{19}$ Even though the complete pulse duration is about $100 \mathrm{~ms}$, we decide to talk of magnetic field pulses of $60 \mathrm{~ms}$ length to consider only the field values higher than $10 \%$ of the maximum field.

${ }^{20}$ F. Duc, J. Vanacken, G. Zhang, W. Decelle, J. E. Lorenzo, C. Detlefs, C. Strohm, T. Roth, R. Suryanarayanan, P. Frings, and G. L. J. A. Rikken, Phys. Rev. B 82, 054105 (2010). 